Revista Calidad en la Educación Superior

Programa de Autoevaluación Académica

Universidad Estatal a Distancia, Costa Rica

ISSN 1659-4703

revistacalidad@uned.ac.cr

\title{
EVALUACIÓN INTERNA DE LA CALIDAD EN LA UNIVERSIDAD DE ATACAMA. HACIA UN MODELO PERMANENTE Y PARTICIPATIVO PARA LA MEJORA CONTINUA
}

\author{
INTERNAL QUALITY EVALUATION OF ATACAMA UNIVERSITY. TOWARDS A \\ MODEL FOR PERMANENT AND PARTICIPATIVE CONTINUOUS \\ IMPROVEMENT
}

Alejandro López-Rodríguez
alelopezrodriguez48@gmail.com
Universidad de Ciencias de la Cultura Física y el Deporte, Cuba
Viviana Lázara González-Maura ${ }^{2}$
psicolo.2011@gmail.com
Universidad de La Habana, Cuba
Jorge Eduardo Valdivia-Díaz
jorge.valdivia@uda.cl
Universidad de Atacama, Chile

Volumen 7, Número 2

Noviembre 2016

pp.180 - 202

Recibido: 21 de julio, 2016

Aprobado: 28 de octubre, 2016

${ }^{1}$ Profesor Titular. Profesor Consultante. Doctor en Ciencias Pedagógicas por el Instituto Central de Ciencias Pedagógicas ICCP. Experto en evaluación externa, certificado por la Junta de Acreditación Nacional JAN de Cuba. Profesor Invitado Universidad de Atacama, Copiapó, Chile (2014-2016). Especialista en currículo y docencia universitaria. Presidente del Comité Editorial de ACCIÓN, Revista cubana de Cultura Física y Miembro de la Junta de Acreditación Nacional de Cuba (2000-2014).Correo electrónico: alelopezrodriguez48@gmail.com

2 Profesora Titular. Doctora en Ciencias Psicológicas y Licenciada en Psicología por la Universidad de La Habana. Miembro del Comité Científico de la Revista Iberoamericana de Educación del Centro de Altos Estudios Universitarios de la Organización de Estados Iberoamericanos (CAEU/OEI), de la Revista de Formación e Innovación Educativa Universitaria (REFIEDU) de la Universidad de Vigo, España y del Consejo Editorial de la Revista Cubana de Educación Superior. Especialista en Motivación y Orientación Profesional, Formación docente universitaria e Investigación Educativa. Profesora Invitada Universidad de Atacama, Copiapó, Chile (2014-2016). Correo electrónico: psicolo.2011@gmail.com

${ }^{3}$ Profesor Asistente. Doctor en Ciencias de la Cultura Física. Profesor de Educación Física. Vicerrector Académico de la Universidad de Atacama. Correo electrónico: jorge.valdivia@uda.cl 


\section{Resumen}

En este artículo se presenta el Modelo de Evaluación Interna de la calidad de la Universidad de Atacama (MEIC-UDA) como herramienta científico-metodológica para el aseguramiento continuo de la calidad. A partir de la concepción de la evaluación interna de la calidad como proceso permanente, participativo, científico y contextualizado, se argumentan y describen sus principios teóricos, ejes de funcionamiento y estructura organizativa. La puesta en práctica del modelo propicia el desarrollo de una cultura de la autoevaluación en la comunidad universitaria, al mismo tiempo que estimula la autorregulación y garantiza la obtención de evidencias objetivas y confiables de la mejora.

Palabras clave: Autoevaluación; Calidad de la educación; Investigación educativa; Modelo.

\section{Abstract}

This scientific article presents the Internal quality Evaluation Model of Atacama University (MEIC-UDA) as a scientific-methodological tool for the continuous quality assurance. The argumentation and description of theoretical principles, operating axes and organizational structure arise from internal quality evaluation conception as a permanent, participative, scientific and contextualized process. This Model implementation allows the development of an auto evaluation culture inside the university, and at the same time, stimulates the autoregulation, ensuring objective and reliable evidences in a sustainable improvement.

Keywords: self-evaluation; quality of education; educational research; model.

\section{Introducción}

La búsqueda de la calidad y su evaluación constituye una proyección asumida en el contexto universitario para el cambio y la transformación. En la actualidad, el debate internacional en torno a la evaluación de la calidad y acreditación institucional y de programas se centra, más que en su necesidad y conveniencia, en el cómo hacerla más efectiva y pertinente en correspondencia con las necesidades de las universidades y las exigencias del contexto social. 
Alejandro López-Rodríguez, Viviana Lázara-González-Maura, Jorge Eduardo Valdivia-Díaz

Los procesos de acreditación que se desarrollan actualmente a escala mundial y, de manera particular, en universidades chilenas, así como su impacto social, constituyen aspectos estratégicos para la mejora continua de la calidad universitaria, en tanto conciben "la evaluación en general y la autoevaluación en particular, como mecanismos de gestión de la calidad" (CNA-Chile, 2008, p.3). Es importante destacar que la autoevaluación universitaria entendida como proceso permanente y dinámico de recogida y análisis de información relevante para la toma de decisiones respecto a la mejora, a partir de estándares y criterios de evaluación establecidos por las agencias de acreditación, que se realiza con la participación y el compromiso de todos los miembros de la comunidad universitaria: académicos, autoridades y directivos constituye un elemento imprescindible en todo proceso de aseguramiento de la calidad (Hernández-Pina, 2014).

Sin embargo, independientemente de que en los documentos normativos para la acreditación, tanto a nivel internacional como en el contexto chileno, se destaca la necesidad e importancia de la autoevaluación como proceso permanente y participativo, se observa una tendencia en la comunidad universitaria a realizar la autoevaluación de forma esporádica, limitada, fundamentalmente al momento previo a la evaluación externa y la acreditación con la que no todos los académicos se sienten comprometidos (CINDA, 2012) (CNA-Chile, 2008).

Esta situación exige centrar la atención en el diseño de modelos de evaluación interna de la calidad en las universidades de manera que constituyan verdaderos procesos de autorregulación orientados a la mejora continua de la formación de los profesionales que en ella tienen lugar a partir de las necesidades internas y del entorno social.

La Universidad de Atacama, con sede en Copiapó, Chile, ha participado en cuatro 
procesos de acreditación institucional. El último se realizó en el 2013 en las áreas obligatorias de Gestión Institucional, Docencia de Pregrado y en el área electiva de Vinculación con el Medio. En esta ocasión, la evaluación externa logró evidenciar un conjunto de fortalezas que le permitieron obtener una acreditación de 4 años, la mayor obtenida hasta ese momento, lo que la ubica dentro del concepto de universidades chilenas consideradas "con mecanismos de aseguramiento de calidad consolidados" (Lemaitre, Maturana, Zenteno y Alvarado, 2012, p.14)

La no obtención de una acreditación de 5 o más años, aspiración declarada en el Plan de Desarrollo Estratégico PDE 2015-2019 (UDA, 2015), estuvo marcada por debilidades detectadas en el proceso de evaluación externa entre las que se destacan: altas tasas de deserción y bajas tasas de titulación, excesivos tiempos de egreso y titulación, insuficiente número de carreras acreditadas y la necesidad de avanzar en la actividad investigativa que se traduzca en un fortalecimiento de la docencia, entre otras.

Con el propósito de cumplir con los objetivos declarados en el PDE 2015-2019 de erradicar las debilidades señaladas en la última acreditación institucional, y potenciar acciones de monitoreo y mejora continua, las autoridades universitarias se plantearon la necesidad de perfeccionar los procesos de autoevaluación. Para llevar a cabo esta misión, se constituyó en septiembre de 2014, con la asesoría de expertos externos, el GEIC (Grupo de Evaluación Interna de la Calidad) cuya tarea consistió en concebir un modelo institucional de evaluación interna para el aseguramiento de la calidad universitaria en la UDA.

En este artículo se presentan los sustentos teóricos y metodológicos del Modelo de Evaluación Interna de la Calidad MEIC-UDA concebido como herramienta científica para la mejora continua de la calidad universitaria. 


\section{- Calidad, Evaluación y Modelo}

Diferentes autores abordan el estudio de la calidad y su evaluación en contextos universitarios (Porto y Mosteiro, 2014) (Lemeitre, et al., 2012) (Román, 2011) (Álvarez, 2009) (Díaz, 2008) (CINDA, 2007) (González, 2006) (Buendía, 2003) (De Miguel, 1996) los que destacan el carácter polisémico del concepto. Es así que la calidad es entendida, en algunos casos, como lo excepcional, lo distintivo, de superior jerarquía, lo perfecto, aquello que no presenta errores. En otros, sin embargo, se refiere a lo que cumple con el propósito para el que fue creado, Fitness for purpose (pertinencia del logro en función del objetivo).

Es interesante destacar que la definición Fitness for Purpose entendida como propósitos establecidos por la propia institución es criticada por Lester (como se citó en Lemaitre, et al., 2012) teniendo en cuenta que, si bien es la más utilizada, resulta limitada al no considerar los requerimientos y objetivos del medio externo. En este sentido, propone sustituirla por fitness of purpose, que incluye el ajuste de los propósitos declarados por la institución a las exigencias pertinentes del medio externo.

En el modelo de evaluación interna de la calidad MEIC-UDA, se entiende por calidad aquello que cumple con el propósito para el que fue creado, propósito que se ajusta a las necesidades internas y a las exigencias pertinentes del medio externo, teniendo en cuenta la distancia que media entre el nivel real de logro constatado y el fin u objetivo que se desea lograr. La calidad así entendida, se asocia al concepto de transformación, como proceso cíclico de mejora continua, centrada en los propósitos y objetivos institucionales, sus principios y prioridades (consistencia interna) y la organización de sus recursos y procesos hacia el cumplimiento de los requerimientos, los estándares de calidad consensuados por la comunidad académica, y las expectativas de los procesos universitarios a nivel social (consistencia externa) (CNAP, 2007). 
Evaluación interna de la calidad en la Universidad de Atacama. hacia un modelo permanente y participativo para la mejora continua.

Alejandro López-Rodríguez, Viviana Lázara-González-Maura, Jorge Eduardo Valdivia-Díaz

Con respecto a la evaluación de la calidad universitaria, se observa un consenso a nivel internacional en cuanto a la necesidad de concebirla como un proceso que integre la evaluación interna y externa.

Es así que en la Declaración Mundial sobre la Educación Superior en el Siglo XXI: UNESCO 1998, se señala que “....una autoevaluación interna y un examen externo realizados con transparencia por expertos independientes, en lo posible especializados en lo internacional, son esenciales para la mejora de la calidad..." (UNESCO, 1998, p. 265)

La Comisión Nacional de Acreditación, CNA-Chile, establece 3 etapas en el proceso de evaluación conducente a la acreditación: la autoevaluación o evaluación interna, realizada por la propia institución; la evaluación externa, realizada por la visita de pares evaluadores; y el pronunciamiento de acreditación (CNA-Chile, 2008). Independientemente de que un objetivo esencial de la acreditación institucional es fortalecer la capacidad de autorregulación de las instituciones de educación superior, en la práctica se observa una tendencia a priorizar la evaluación externa en detrimento de la autoevaluación en la evaluación de la calidad universitaria (Venables y Van Gastel, 2014)

La evaluación de la calidad en el MEIC- UDA se concibe como un proceso investigativo riguroso y permanente, mediante el cual se ofrece información objetiva, pertinente y relevante para la toma de decisiones institucionales, orientadas a la mejora continua de la calidad universitaria. En este sentido, defiende la idea de que el propósito esencial de una evaluación interna de la calidad universitaria no es demostrar, sancionar o validar, sino hacer emerger el conocimiento y los aprendizajes, que hagan posible reorientar y mejorar lo planificado e implementado, a fin de conseguir los resultados y efectos buscados y requeridos. 
Lo anterior significa usar la evaluación "como un proceso de reflexión valorativa y sistemática que contribuye decididamente a la mejora de la calidad..." Ruiz (como se citó en González, 2006, p.15).

En cuanto al concepto de Modelo, Sacristán (1985) lo define como un esquema mediador entre la realidad y el pensamiento, entre el mundo y la ciencia, el filtro de la información que buscamos de la realidad, una estructura en torno a la que organizar el conocimiento, una fuente de hipótesis de investigación, un recurso, en definitiva, imprescindible para el desarrollo de la ciencia. Piola (2000) desde una perspectiva dialéctica destaca la concepción dinámica del modelo en tanto proceso abierto, inacabado, permanente, en constante diálogo con realidades cambiantes, que permite reconstruirlo a partir de las nuevas evidencias empíricas. En ambos casos, se destaca en la concepción del modelo el necesario nexo entre la teoría y la práctica, es por ello que todo modelo tiene un valor teórico y metodológico, toda vez que no sólo permite representar y explicar la realidad a través de categorías, principios, regularidades, sino también constituye una guía para su transformación.

Buendía (2003) en el artículo "Hacia una universidad de calidad: algunos problemas pendientes" identifica dos modelos de evaluación de la calidad universitaria, los que denomina: racional-burocrático y contextual-comunitario.

Estos modelos tienen dos claros posicionamientos teóricos: el primero, basado en teorías psicológicas conductistas centrado en los input y output, mientras que el segundo, se sustenta en teorías psicológicas del constructivismo social centrado en los sujetos, las interacciones y el contexto.

El modelo racional-burocrático, también denominado tradicional, concibe las instituciones educacionales como organizaciones formales, privilegia el 
rendimiento y el resultado, o producto, como sinónimos de calidad, en detrimento del proceso desarrollado para su consecución. Las instituciones que conciben la evaluación de la calidad universitaria desde este modelo centran la atención en la evaluación externa y la acreditación, por lo que, el aseguramiento de la calidad al interior de las universidades se realiza a través del establecimiento de mecanismos institucionales de planificación y control, que garanticen el cumplimiento de las exigencias de los procesos de acreditación, al margen de la participación y el compromiso de los principales actores: académicos y estudiantes.

La autoevaluación en este modelo se caracteriza por ser asistemática si tenemos en cuenta que la información en que se sustenta el informe de evaluación interna se obtiene en un período previo a la solicitud de evaluación externa de pares o agencias evaluadoras. Por otra parte, su representatividad es parcial ya que se realiza fundamentalmente por las estructuras de dirección con baja o nula participación del claustro, con evaluaciones respaldadas por análisis linealescausales. El fin último de este modelo es, por tanto, identificar y clasificar las instituciones en centros "más eficaces" y "menos eficaces" a partir de resultados eminentemente cuantitativos.

El modelo contextual-comunitario, a diferencia del anterior, centra la atención en el proceso y la interacción entre los miembros de la comunidad educativa, como elementos esenciales en la evaluación de la calidad.

Este modelo concibe la Universidad como una comunidad interactiva de miembros, "pequeñas sociedades o ecosistemas" (Buendía, 2003, p.119) o comunidad universitaria integrada por académicos, investigadores, estudiantes, funcionarios y personal de apoyo, entre otros. Centra la atención en la interacción permanente entre el micro y macro sistema: por una parte, entre los miembros de 
la comunidad universitaria en las aulas, laboratorios, terrenos, escenarios, reales 0 simulados, de prácticas laborales, y por otra, entre la comunidad universitaria y la sociedad en general, tanto a nivel regional, nacional o internacional. (Aguilera, Mendoza, Racionero y Soler, 2010). El contexto y la participación de la comunidad universitaria, por tanto, se convierten en las variables diferenciadoras, a partir de las cuales se realiza el análisis y la búsqueda de alternativas de solución a los problemas identificados en las asignaturas, carreras, facultades o Universidad, teniendo en cuenta las metas y estándares establecidos para la mejora de la calidad.

El modelo de evaluación interna de la Calidad de la UDA que se presenta asume como paradigma para el aseguramiento de la calidad de los procesos universitarios, el modelo contextual-comunitario, tomando en cuenta que la evaluación interna de la calidad universitaria debe realizarse a través de la participación activa y comprometida de todos sus miembros en correspondencia con las necesidades y exigencias del contexto social.

\section{- El Modelo de Evaluación Interna de la calidad de la UDA (MEIC-UDA)}

EI MEIC-UDA tiene como objetivo: Perfeccionar el proceso de Autoevaluación institucional a través de la utilización de la investigación científica y la participación activa y comprometida de todos los miembros de la comunidad universitaria en la mejora continua de la calidad.

Se orienta, por tanto, a instalar en cada miembro de la comunidad universitaria una cultura de la evaluación como proceso de mejora continua, atemperada a su jerarquía académica y/o el rol académico, administrativo o de servicio que realiza. La evaluación interna de la calidad en este modelo se define como un proceso de evaluación permanente, sistémico, participativo y contextualizado sustentado en la investigación científica que permite mostrar evidencias objetivas y confiables de la 
Evaluación interna de la calidad en la Universidad de Atacama. hacia un modelo permanente y participativo para la mejora continua.

Alejandro López-Rodríguez, Viviana Lázara-González-Maura, Jorge Eduardo Valdivia-Díaz

mejora continua de la calidad universitaria.

\section{La evaluación interna como proceso permanente.}

La evaluación interna se concibe como un proceso continuo de identificación de los problemas que afectan la calidad universitaria, a partir del cual se diseñan planes de acción con objetivos a corto, mediano y largo plazo dirigidos a la búsqueda de alternativas para su solución. De esta manera la autoevaluación deja de ser el punto de partida de la evaluación externa y la acreditación al convertirse en un estilo de trabajo permanente que permite a la comunidad universitaria trabajar de forma sistemática en la mejora de la calidad de sus procesos.

\section{La evaluación interna como proceso sistémico.}

La evaluación interna de la calidad universitaria es un proceso sistémico, si tenemos en cuenta que las acciones de mejora que se realizan en las diferentes áreas universitarias: docencia, investigación, vinculación con el medio, y gestión institucional, están íntimamente relacionadas. Por tanto, todo cambio que se introduzca en un área tiene su impacto en las restantes. Es por ello que no es posible trabajar por la mejora en las diferentes áreas y procesos universitarios de forma aislada porque constituyen un sistema.

\section{La evaluación interna como proceso participativo.}

La evaluación interna de la calidad universitaria es un proceso eminentemente participativo ya que son los miembros de la comunidad universitaria los protagonistas del cambio que de manera conjunta identifican los problemas que afectan la calidad y reflexionan en torno a cómo encontrar las mejores y posibles alternativas para su solución. (Tünnermann, 2008). 
Evaluación interna de la calidad en la Universidad de Atacama. hacia un modelo permanente y participativo para la mejora continua.

Alejandro López-Rodríguez, Viviana Lázara-González-Maura, Jorge Eduardo Valdivia-Díaz

\section{La evaluación interna como proceso contextualizado.}

La evaluación interna ha de dar respuesta a los problemas que afectan la calidad universitaria en el contexto en que éstos se producen. Es por ello que los planes de acción para la mejora, necesariamente, tienen que elaborarse teniendo en cuenta las características, exigencias, potencialidades, limitaciones y oportunidades de los contextos en que se desarrollan.

\subsection{Principios que orientan la implementación del modelo.}

La implementación del modelo de evaluación interna de la calidad UDA se sustenta en 6 principios. Ellos son:

- El carácter científico de la autoevaluación

- La unidad entre diagnóstico e intervención.

- La flexibilidad en la implementación del modelo de evaluación interna.

- El compromiso con el cambio y la mejora.

- El carácter formativo de la autoevaluación.

- La unidad entre la evaluación interna y externa.

\section{El carácter científico de la autoevaluación.}

La utilización sistemática de la investigación científica como herramienta metodológica tanto en el diagnóstico de los problemas que afectan la calidad universitaria, como en el diseño, implementación y evaluación de las acciones orientadas a su solución, permite mostrar evidencias objetivas y confiables de los cambios logrados en la mejora de la calidad, es por ello que la investigación científica para la mejora de los procesos universitarios se constituye en un eje transversal del modelo de evaluación interna de la calidad.

\section{La unidad del diagnóstico y la intervención.}

Teniendo en cuenta que se parte de un concepto de evaluación con fines de transformación, (Yarzábal, 1999) el diagnóstico constituye el punto de partida 
necesario para el diseño de acciones interventivas dirigidas a la mejora de la calidad en la educación universitaria. No obstante, por el carácter continuo de la autoevaluación el diagnóstico no sólo precede sino que acompaña las acciones de intervención con propósitos de control y evaluación sistemática.

\section{La flexibilidad en la implementación del modelo de autoevaluación.}

Al tener la evaluación interna de la calidad universitaria un carácter permanente y contextualizado, debe ser flexible de manera que pueda adecuarse, no sólo a las características de los diferentes escenarios de aplicación, sino también a los cambios que gradualmente se van logrando en el proceso de su implementación.

\section{El compromiso con el cambio y la mejora.}

El compromiso de todos los miembros de la comunidad universitaria con el cambio es un principio esencial en la autoevaluación orientada a la mejora de la calidad en las instituciones de educación superior (Venables y Van Gastel, 2014) (Santos, 1995, 2001, 2010) (De Miguel y Apodaca, 2009) (Álvarez, 1997, 2009) (Díaz, 2008) (De Miguel, 1996). El cambio sólo es posible cuando los participantes se comprometen, y por tanto, trabajan en conjunto por la mejora de la calidad de su desempeño.

\section{El carácter formativo de la autoevaluación.}

El proceso de evaluación interna de la calidad se concibe como un proceso formativo, toda vez que al sustentarse en la reflexión individual y grupal de los miembros de la comunidad universitaria en la búsqueda de alternativas para la mejora se potencia su desarrollo profesional y personal (Santos, 2010).

\section{La unidad entre la evaluación interna y externa.}

En el modelo que se presenta, la autoevaluación es concebida como un proceso permanente de reflexión y diálogo entre los miembros de la comunidad 
universitaria, orientado a la mejora continua de la calidad universitaria que exige la acreditación. Por tanto, ambos procesos, evaluación interna y externa constituyen una unidad, toda vez que la evaluación interna es al mismo tiempo punto de partida y resultado de la acreditación en el proceso de mejora continua de la calidad universitaria que funciona como una espiral de desarrollo (Tünnermann, 2008) (Álvarez, 2009).

\subsection{Ejes de funcionamiento del modelo.}

Teniendo en cuenta el carácter científico, participativo y formativo del modelo institucional de evaluación interna de la calidad UDA, su funcionamiento se desarrolla en torno a tres ejes centrales:

- La investigación científica para la mejora de la calidad de los procesos universitarios.

- La unidad entre autoevaluación, coevaluación y heteroevaluación en el proceso de evaluación interna.

- La formación permanente del claustro en docencia, gestión e investigación universitaria.

\section{La investigación científica para la mejora de la calidad de los procesos universitarios.}

La investigación científica para la mejora de la calidad de los procesos universitarios, eje principal del modelo de evaluación interna de la calidad UDA, reconoce la necesidad y posibilidad de utilizar de forma independiente, 0 combinada, diferentes paradigmas de investigación (Clásico, Interpretativo, Crítico), mediante diseños mixtos, cualitativo - cuantitativos, aunque privilegia la utilización del paradigma Crítico, (Diez, 2013) (González, Blández, López y Sierra, 2007) (Blández, 1996) (Arnal, 1992) (Carr, 1990) (Carr y Kemmis, 1988) (Stenhause, 1987) que se orienta no sólo a la explicación o comprensión de los problemas identificados, sino fundamentalmente a su transformación. Es por ello 
que la investigación-acción constituye la herramienta esencial del modelo.

El modelo asume la investigación-acción como método de investigación y estrategia formativa integral y de aprendizaje colaborativo en todos los niveles institucionales, y para todos los miembros de la comunidad universitaria que forman parte de los grupos de investigación: directivos, académicos y especialistas.

\section{La unidad entre autoevaluación, coevaluación y heteroevaluación en el proceso de evaluación interna.}

La unidad entre autoevaluación, coevaluación y heteroevaluación en el proceso de evaluación interna constituye otro eje esencial en la labor docente investigativa, extensionista y de gestión que desarrolla cada académico y las diferentes instancias universitarias. De esta manera, ser parte de la reflexión crítica y autocrítica de cada miembro de la comunidad universitaria acerca de cómo está trabajando, para la mejora de la calidad la cual es enriquecida por la valoración de otros miembros con los que comparte su quehacer en la institución.

\section{La formación permanente del claustro en docencia, gestión e investigación universitaria.}

Dado el carácter participativo del MEIC-UDA, los académicos y directivos constituyen el principal recurso humano para el cambio y la mejora de la calidad. En tal sentido, resulta esencial el diseño e implementación de un sistema de capacitación y actualización permanente en temas relacionados con la docencia, la gestión y la investigación universitaria, que propicien el desarrollo de las competencias necesarias para el trabajo por la mejora continua de la calidad.

\section{- Estructura organizativa del modelo de evaluación interna de la calidad UDA.}

La implementación del modelo se concreta en diferentes estrategias, acciones y 
formas evaluativas a través de la constitución de las siguientes estructuras organizativas: el Comité de Evaluación Interna de la Calidad (CEIC) y los Grupos Ad-Hoc. EI CEIC es la máxima estructura organizativa para la evaluación interna de la calidad. Este comité se conforma como un equipo multidisciplinario de especialistas en docencia, investigación, gestión y extensión universitaria, que realiza funciones de coordinación, asesoría y capacitación a los miembros de los grupos Ad-hoc en Evaluación e Investigación para la mejora de la calidad universitaria. Fig.1

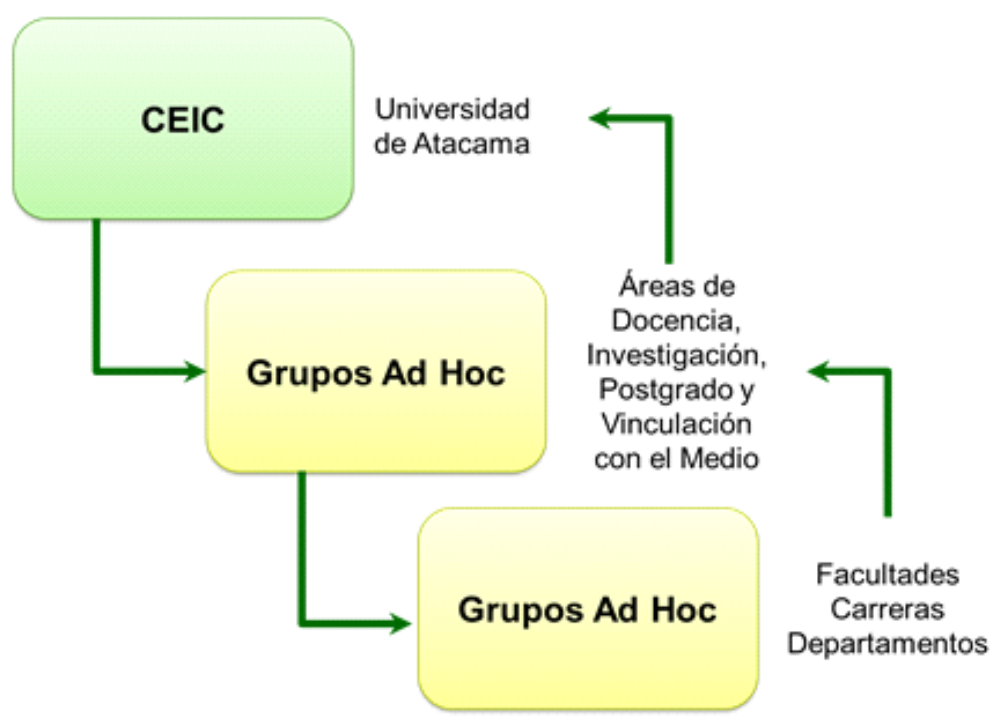

Fig. 1. Interacción CEIC - Grupos Ad - Hoc

Conceptualmente, un Grupo Ad-hoc en este modelo es un equipo de investigación que se conforma para profundizar en el estudio de problemas específicos, previamente identificados, que afectan la calidad de los procesos universitarios. Constituye la estructura base organizativa del modelo en las distintas instancias de dirección (Facultades, Carreras) y/o en distintas Áreas (Pregrado, Investigación, Gestión Institucional y Vinculación con el Medio), con el objetivo de desarrollar proyectos de investigación, innovación educativa o planes de acción para la 
Evaluación interna de la calidad en la Universidad de Atacama. hacia un modelo permanente y participativo para la mejora continua.

Alejandro López-Rodríguez, Viviana Lázara-González-Maura, Jorge Eduardo Valdivia-Díaz

mejora de la calidad que involucren progresivamente a otros miembros de la comunidad universitaria.

Un requisito de la constitución de los Grupos Ad-hoc es la participación voluntaria de sus miembros, lo que favorece mejores niveles de adhesión y compromiso con el trabajo por la mejora.

La intervención de los propios miembros de los Grupos Ad-hoc en el análisis reflexivo y crítico de la realidad institucional posibilita un acercamiento entre los diferentes sectores institucionales, un mayor conocimiento y dimensionamiento de las situaciones, una visión compartida de las problemáticas y una búsqueda conjunta, interactiva y comprometida de soluciones y propuestas que conduzcan a la mejora, y ello resulta vital, dada la esencia contextual- comunitaria del modelo.

A diferencia de otras formas de organización social, en los Grupos Ad-hoc se establece una relación sujeto-sujeto a partir de un diálogo democrático en el cual se busca la reflexión crítica sobre la realidad y su transformación a través de acciones concretas.

\section{Etapas del modelo de evaluación interna de la calidad UDA.}

Tal y como se definió anteriormente, la evaluación interna de la calidad universitaria se concibe como un proceso de evaluación permanente, sistémico, participativo y contextualizado orientado a la mejora continua de la calidad, sustentado en la utilización de la investigación científica y el uso de las TIC.

Como todo proceso sistemático exige seguir un conjunto de pasos los cuales se integran en 6 etapas.

- 1era etapa. Diagnóstico y construcción participativa del modelo de evaluación interna de la calidad.

- 2da etapa: Socialización e implantación del modelo.

- 3era etapa: Implementación de planes de acción del CEIC y desarrollo de 
proyectos de investigación por los Grupos Ad-Hoc.

- 4ta etapa: Elaboración del informe de Autoevaluación.

- 5ta etapa: Evaluación externa de pares.

- 6ta etapa: Reconstrucción participativa del modelo.

Las etapas tienen un carácter cíclico y en espiral. Cíclico porque abarca un proceso de investigación y mejora, que comprende 3 etapas asociadas con todo el proceso de construcción, socialización, implantación e implementación, 2 etapas donde se plasman los resultados en el informe de autoevaluación y se somete a evaluación externa de pares; y en espiral, porque a partir de los resultados de la quinta etapa, se genera una sexta que contempla un nuevo proceso de reconstrucción del modelo, que implica mantener y consolidar todo aquello que se ha justificado por su pertinencia y eficacia, e introducir nuevos conceptos $y$ procedimientos que permitan mantener lo logrado y proyectarse hacia nuevas metas.

\section{Convergencia entre el DAC (Dirección de Aseguramiento de la Calidad) y el MEIC (Modelo de Evaluación Interna de la Calidad) en el proceso de autoevaluación institucional y de carreras.}

La Universidad de Atacama mediante el decreto exento № 435 creó la Dirección de Aseguramiento de la Calidad (DAC), la cual entra en operación en noviembre de 2011. Esta estructura administrativa centra su labor en mantener fluidos canales de comunicación con la CNA, gestionar los procesos institucionales destinados al mejoramiento continuo de las funciones universitarias, optimizar los resultados alcanzados en la acreditación institucional y de carreras así como, obtener información relevante para preparar informes de autoevaluación institucional y realizar mediciones conducentes a la formación de indicadores de resultados, apoyado en facilitadores e interlocutores. 
El Modelo de Evaluación Interna de la Calidad MEIC, aprobado por el Consejo Académico en Enero de 2015, por su parte, ofrece a la comunidad universitaria las herramientas metodológicas necesarias para el desarrollo de un proceso científico y participativo de mejora continua de la calidad que aporte al informe de autoevaluación, que exige la evaluación externa, evidencias objetivas y confiables de los resultados obtenidos en el trabajo por la mejora de indicadores de eficiencia en las diferentes facultades, carreras e instancias universitarias. Fig. 2

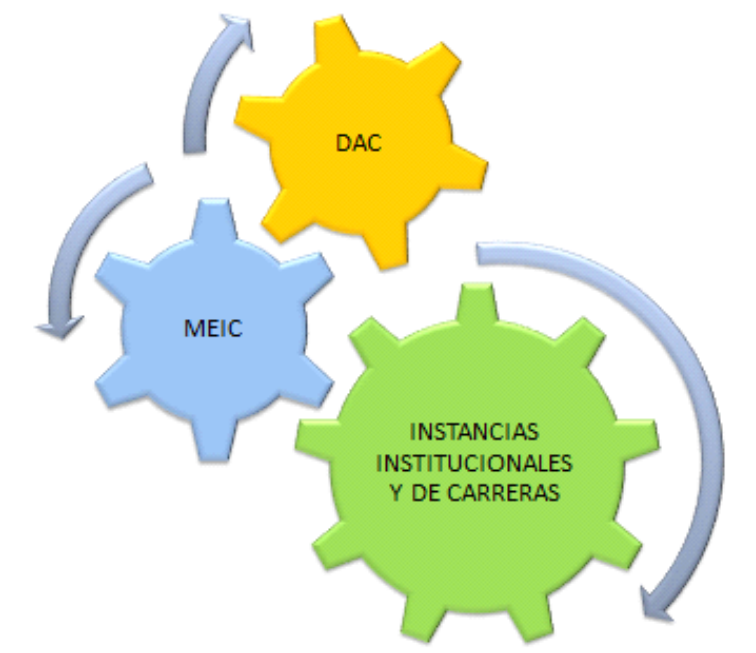

Fig. 2. Mecanismos de autoevaluación institucional y de carreras en la UDA

Tanto el DAC como el MEIC, hacen un amplio uso de las Tecnologías de la Información y la Comunicación TIC en el desarrollo de la evaluación interna de la calidad. El primero, a través de un sistema computarizado de control y gestión (SCG), para el monitoreo del Plan de Desarrollo Estratégico y un sistema de información integral de datos, y el segundo, mediante la utilización de páginas web, para la comunicación e intercambio entre los miembros de la comunidad universitaria y de portafolios electrónicos (Campaña, 2013) para el registro de las evidencias de mejora. 
Alejandro López-Rodríguez, Viviana Lázara-González-Maura, Jorge Eduardo Valdivia-Díaz

La conjugación efectiva del trabajo de ambas estructuras, una administrativa y otra funcional, favorece un abordaje integral, permanente y participativo en la mejora continua de la calidad universitaria.

\section{Conclusiones}

El modelo de evaluación interna de la calidad UDA, como se evidencia en el artículo, constituye una aplicación teórica y metodológica del modelo contextual comunitario de evaluación de la calidad universitaria a la concepción y práctica de la autoevaluación institucional en la Universidad de Atacama.

La novedad e importancia del modelo presentado estriba en su capacidad de promover una amplia participación y compromiso de los académicos, autoridades y directivos en el trabajo por la mejora continua de la calidad a través de la investigación científica y el trabajo colaborativo. Al mismo tiempo, genera una cultura de autoevaluación y formación permanente en el ejercicio de la docencia y la gestión universitaria.

El modelo de evaluación interna de la calidad UDA en tanto constituye una herramienta científica para la autoevaluación institucional, permite obtener evidencias objetivas y confiables de la mejora continua de la calidad universitaria necesarias para el logro de niveles superiores de acreditación. 


\section{Referencias}

Aguilera, A., Mendoza, M., Racionero, S. y Soler, M. (2010). El papel de la universidad en comunidades de aprendizaje. Revista interuniversitaria de formación del profesorado, 67(24,1), 45-56. Recuperado de http://m.aufop.com/aufop/uploaded files/articulos/1268700514.pdf

Álvarez, J. (1997). La autoevaluación institucional en los centros educativos: una propuesta para la acción. Madrid: Universidad Complutense.

Álvarez, J. (2009). Evaluación institucional: entre las urgencias políticas y las necesidades de mejora de los centros. Organización y gestión educativa: Revista del Fórum Europeo de Administradores de la Educación, 67(24,1), $21-25$.

Arnal, J. (1992). Investigación educativa. Fundamentos y metodología. Barcelona: Labor.

Blandez, J. (1996). La investigación - acción. Un reto para el profesorado. Barcelona: INDE.

Buendía, L. (2003). Hacia una universidad de calidad: algunos problemas pendientes. Universidad de Granada. Educatio, pp. 20-21, Dic. Recuperado de http://revistas.um.es/educatio/article/viewFile/135/119

Campaña, K. (2013). El portafolio virtual como herramienta para la práctica reflexiva. Valoración en su empleo en el practicum. Tesis doctoral. Universidad de Navarra, España.

Carr, W. (1990). Hacia una ciencia crítica de la educación. Barcelona: Laertes.

Carr, W. y Kemmis, S. (1988). Teoría crítica de la enseñanza. La investigaciónacción en la Formación del profesorado. Barcelona: Martínez Roca.

CINDA (2012). Aseguramiento de la calidad en Iberoamérica. Educación Superior. Informe $2012.2 \quad$ Recuperado de http://www.universia.net/nosotros/files/CINDA-2012-Informe-deEducaci\%C3\%B3n-Superior.pdf

CINDA (2007). Evaluación del desempeño docente y calidad de la docencia universitaria. MINEDUC-Chile. Recuperado de: http://www.cinda.cl/download/libros/EVALUACl\%C3\%93N\%20DEL\%20DES 
Evaluación interna de la calidad en la Universidad de Atacama. hacia un modelo permanente y participativo para la mejora continua.

Alejandro López-Rodríguez, Viviana Lázara-González-Maura, Jorge Eduardo Valdivia-Díaz

\section{EMPE\%C3\%910\%20DOCEN.pdf}

CNA-Chile (2008). Manual para el desarrollo de procesos de autoevaluación. Carreras y programas de pregrado. Comisión Nacional de Acreditación, CNA-CHILE. Secretaría Ejecutiva. Recuperado de: https://www.cnachile.cl/.../Acreditacion\%20Pregrado/.../Manual Autoevaluacion.pdf

CNAP (2007). El modelo chileno de acreditación de la educación superior. Santiago, Chile. Recuperado de: www.cieucinf.cl/download/.../CIE mod chileno acreditacion.pdf

De Miguel, M. (1996). La evaluación de las instituciones universitarias, en: Documentos Didácticos. (157). IUCE. Salamanca.

De Miguel, M. y Apodaca, P. (2009). Aseguramiento versus garantía de calidad en el sistema universitario español. Boletín de Psicología, 97, 35-54. Recuperado de http://www.uv.es/seoane/boletin/previos/N97-3.pdf

Díaz, J. (2008). Calidad, pertinencia y responsabilidad social de la universidad latinoamericana y caribeña. En: A. L. Gazzola y A. Didriksson (Eds.). Tendencias de la Educación Superior en América Latina y el Caribe, pp.87112 Caracas: IESALC-UNESCO.

Diez, E.J. (2013). Investigación-acción participativa: el cambio cultural con la implicación de los participantes. Revista Electrónica Interuniversitaria de Formación del Profesorado, 16(3), 115-131. doi: http://dx.doi.org/10.6018/reifop.16.3.186171

González, I. (2006). Dimensiones de evaluación de la calidad universitaria en el Espacio Europeo de Educación Superior. Revista electrónica de investigación psicoeducativa, 10,4(3), 445-468, Recuperado de http://www.investigacionpsicopedagogica.org/revista/articulos/10/espannol/Art 10 134.pdf

González, V., Blández, J., López, A. y Sierra, M.Á. (2007). Educar la responsabilidad profesional a través de la investigación-acción. Una experiencia en la formación del profesorado en educación física. Revista de 
investigación educativa. Tiempo de Educar, 8(15), 113-138. Recuperado de http://www.redalyc.org/pdf/311/31181505.pdf

Lemaitre, M. J., Maturana, M., Zenteno, E. y Alvarado, A. (2012). Cambios en la gestión institucional en universidades, a partir de la implementación del Sistema Nacional de Aseguramiento de la Calidad: la experiencia chilena. Calidad en la Educación (36), Santiago de Chile. Recuperado de http://www.scielo.cl/pdf/caledu/n36/art01.pdf

Hernández-Pina, F. (2014). Evaluación y acreditación del profesorado, programas e instituciones educativas. Revista Electrónica Interuniversitaria de Formación del Profesorado,17(1), 15-32. Recuperado de http://dx.doi.org/10.6018/reifop.17.1.198821

Piola, M. (2000). Paradigmas en crisis ante los nuevos y viejos desafíos de la cuestión social en América Latina, en: Scripta Nova. Revista electrónica de Geografía y Ciencias Sociales, Universidad de Barcelona, 69(80). Recuperado de http://www.ub.edu/geocrit/sn-69-80.htm

Porto, A. M. y Mosteiro, M. J. (2014). Innovación y calidad en la formación del profesorado universitario. Revista Electrónica Interuniversitaria de Formación del Profesorado, 17(3), 141-156. doi: http://dx.doi.org/10.6018/reifop.17.3.204101

Román, M. (2011). Autoevaluación: estrategia y componente esencial para el cambio y la mejora escolar, en Revista Iberoamericana de Educación, (55), 107-136, Recuperado de http://rieoei.org/rie55a04.pdf

Sacristán, J. G. (1985). Teoría de la enseñanza y desarrollo del currículo. Madrid: Ed. Araya.

Santos, M. (1995). La evaluación: un proceso de diálogo, comprensión y mejora. Málaga: Aljibe.

Santos, M. (2001). La escuela que aprende. Madrid: Morata.

Santos, M. (2010). La formación del profesorado en las instituciones que aprenden Revista interuniversitaria de formación del profesorado, 68(24.2), 175-200, Recuperado de file:///C:/Users/usuario/Downloads/DialnetLaFormacionDelProfesoradoEnLasInstitucionesQueApre3276058\%20(1).pdf 
Stenhouse, L. (1987). La investigación como base de la enseñanza. Madrid: Morata

Tünnermann, C. (2008). La calidad de la educación superior y su acreditación: la experiencia centroamericana. Avaliação, Campinas; Sorocaba, SP, 13(2), 313-336, Recuperado de http://www.scielo.br/pdf /aval/ v13n2/05pdf.

UNESCO (1998). Declaración Mundial sobre la Educación Superior en el Siglo XXI: Visión y Acción. Conferencia mundial sobre la educación superior UNESCO, París 5-9 de octubre. Recuperado de bvs.sld.cu/revistas/ems/vol14_3_00/ems06300.pdfe

Universidad de Atacama UDA (2015). Plan de Desarrollo Estratégico 2015-2019. Documento no publicado.

Venables, J. C. y Van-Gastel, J. (2014). Radiografía de los modelos de acreditación: organización, procesos y prácticas. El caso de las universidades Austral de Chile, de la Frontera y de los Lagos. Calidad en la Educación, 41, 51-81. Recuperado de www.scielo.cl/pdf/caledu/n41/art03.pdf

Yarzábal, L. (1999). Evaluar para transformar. IESALC-UNESCO. 